MATEC Web of Conferences 13, 04028 (2014)

DOI: $10.1051 /$ matecconf/ 20141304028

(C) Owned by the authors, published by EDP Sciences, 2014

\title{
Experimental Preparation and Numerical Simulation of High Thermal Conductive Cu/CNTs Nanocomposites
}

\author{
Ali Samer Muhsan ${ }^{\mathrm{a}, 1}$, Faiz Ahmad ${ }^{1}$, Norani M. Mohamed ${ }^{2}$, Puteri Sri Melor Megat Yusoff ${ }^{1}$, M. Rafi Raza ${ }^{3}$, \\ and Iman Eslami Afrooz ${ }^{1}$ \\ ${ }^{1}$ Department of Mechanical Engineering, Universiti Teknologi PETRONAS (UTP), Malaysia \\ ${ }^{2}$ Centre of Innovative Nanostructures and Nanodevices (COINN), UTP, Malaysia \\ ${ }^{3}$ Universiti Kebangsaan Malaysia, 43600, Bangi, Selangor, Malaysia
}

\begin{abstract}
Due to the rapid growth of high performance electronics devices accompanied by overheating problem, heat dissipater nanocomposites material having ultra-high thermal conductivity and low coefficient of thermal expansion was proposed. In this work, a nanocomposite material made of copper $(\mathrm{Cu})$ reinforced by multi-walled carbon nanotubes (CNTs) up to 10 vol. \% was prepared and their thermal behaviour was measured experimentally and evaluated using numerical simulation. In order to numerically predict the thermal behaviour of $\mathrm{Cu} / \mathrm{CNTs}$ composites, three different prediction methods were performed. The results showed that rules of mixture method records the highest thermal conductivity for all predicted composites. In contrast, the prediction model which takes into account the influence of the interface thermal resistance between CNTs and copper particles, has shown the lowest thermal conductivity which considered as the closest results to the experimental measurement. The experimentally measured thermal conductivities showed remarkable increase after adding 5 vol.\% CNTs and higher than the thermal conductivities predicted via Nan models, indicating that the improved fabrication technique of powder injection molding that has been used to produced $\mathrm{Cu} / \mathrm{CNTs}$ nanocomposites has overcome the challenges assumed in the mathematical models.
\end{abstract}

\section{Introduction}

The next generation of high performance electronic devices required exceptional packaging materials with special thermal properties such as high thermal conductivity and low coefficient of thermal expansion. High performance heat sink material produced by advanced technique is needed to dissipate the excessive heat generated from next generation electronic devices [1]. Due to its extraordinary physical, chemical and mechanical properties among others, carbon nanotubes (CNTs) have been chosen as the most promising nanomaterial in the field of advanced material [2, 3]. However, due to the existence of some drawbacks, limited studies have dealt with the physical properties of CNTs reinforced metal matrix nanocomposites $[4,5]$. These main drawbacks can be identified as follows: weak interfacial bonding or poor wetting behaviour between CNTs and metal

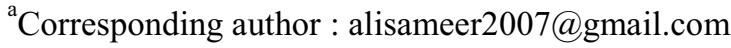


matrix, inhomogeneous distribution of CNTs in the matrices, agglomeration among themselves with Van-der Waals force and degraded thermal stability at processing temperature [6]. However, some researchers have focused on evaluation of thermal conductivity of carbon nanotubes reinforced composite using numerical methods. In 2003, Nan et al. [7] have suggested a simple mathematical model to predict the thermal conductivity behaviour of carbon nanotubes reinforced composites using conventional effective medium method. They reported that dispersion of a quite small amount of CNTs can result in a remarkable enhancement in the effective thermal conductivity of the composites. In 2004, Nan et al. [8] have furtherer modifying their theory by incorporating the influence of interface thermal resistance between CNTs and the matrix composite. They predicted that a large interface thermal resistance across the nanotube-matrix interface causes a significant degradation in the thermal conductivity enhancement, even for the case with ultrahigh intrinsic thermal conductivity and aspect ratio of the carbon nanotubes embedded. In order to improve the thermal conductivity of the fabricated $\mathrm{Cu} / \mathrm{CNT}$ s composites, sufficient information on its thermal behaviour before and after adding the reinforcement is needed. In addition, to determine the effects of other factors that may occur during fabrication processes, such as interfacial thermal resistance between CNTs and matrix particles and CNTs clusters in the matrix. Therefore, this study aims to evaluate the experimentally measured thermal conductivities of $\mathrm{Cu} / \mathrm{CNTs}$ nanocomposites with numerically simulated prediction models using finite element method and compared with some theoretical models that taken in account a uniform dispersion of CNTs inside the $\mathrm{Cu}$ matrices and the influence of the interfacial thermal resistance between $\mathrm{Cu}$ matrix and individual CNT.

\section{Experimental Protocols}

\subsection{Sample Preparation}

The preparation of MWNT/Cu composites has been explained with detail in the previous work $[9,10]$. However, a brief description is of experimentation is provided here. First, functionalization process has been applied to enhance the dispersion of purified MWNTs and creates sidewalls groups (-COOH) that have the ability to bond MWNTs to the $\mathrm{Cu}$ matrix. The incentive for carrying out this step came from the experimental results accomplished by some researchers who dealt with polymer nanocomposites. However, in this experiment, MWNTs were immersed in concentrated acid solution of $\mathrm{H}_{2} \mathrm{SO}_{4}(98 \%)$ and $\mathrm{HNO}_{3}(67 \%)$ prepared in a ratio of 3:1. Each 1gram of MWNTs was mixed with $250 \mathrm{~mL}$ of acid solution and heated up to $70^{\circ} \mathrm{C}$ with sonication for $2.75 \mathrm{~h}$. The treated MWNTs were then washed several times with distilled water and dried at $50^{\circ} \mathrm{C}$ for $12 \mathrm{~h}$. MWNTs were then mixed with low viscous liquid media of dissolved paraffin wax (PW/Heptane) along with magnetic stirring for $1 \mathrm{~h}$. Each 1 gram of functionalized MWNTs was mixed with $250 \mathrm{~mL}$ of PW/Heptane (1:4) solution and agitated for $1 \mathrm{~h}$ using magnetic stirrer. This again was followed by sonication process to ensure uniform dispersion of functionalized MWNTs in PW solution and dried at $50^{\circ} \mathrm{C}$ for $2 \mathrm{~h}$.

\subsection{Numerical Simulation}

The model can be simplified as cylindrical fins having a diameter of $1 \mathrm{~mm}$ and length of $4 \mathrm{~mm}$ attached on a flat square plate of $10 \times 10 \mathrm{~mm}$ in area with thickness of $1 \mathrm{~mm}$ as shown in Figure 1a. The lower surface of the heat sink is submitted to uniform external heat source (electronic chip) of $110^{\circ} \mathrm{C}$. In contrast, the other surface is attached by 49 fins, which conventionally dissipate the heat to the ambient air $40^{\circ} \mathrm{C}$. The inner and outer heat dissipated $\left(\mathrm{Q}_{\text {in }}\right)$ and $\left(\mathrm{Q}_{\text {out }}\right)$ respectively, was assumed to have the same direction of that for temperature (parallel to the fins direction) as illustrated in Fig. 1b. 


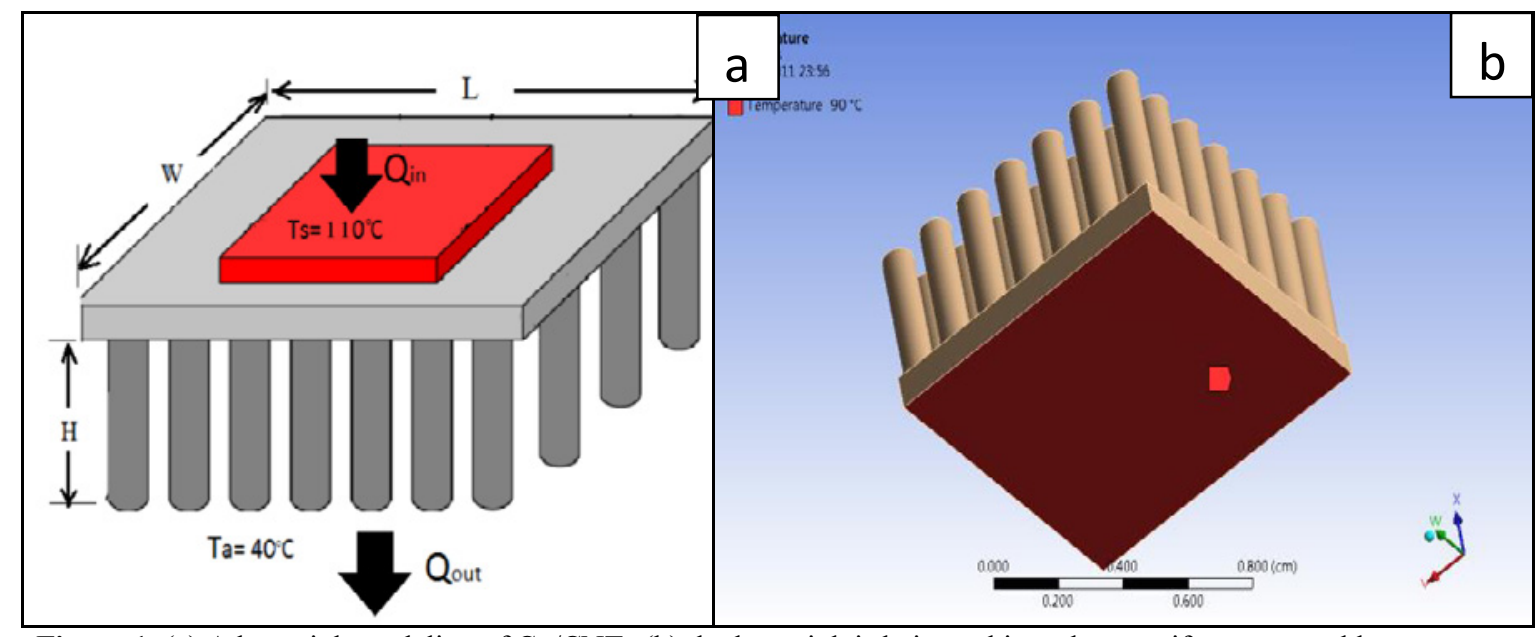

Figure 1. (a) A heat sink modeling of $\mathrm{Cu} / \mathrm{CNTs}$ (b) the heat sink is being subjected to a uniform external heat source (electronic chip) of $110^{\circ} \mathrm{C}$

\subsection{Thermal conductivity measurement of Cu/CNTs Composites}

The model used in present study is a composite material of two components; pure copper as a matrix and CNTs as reinforcement. Four different contents of CNTs 2, 5, 7.5 and 10 vol. \% were incorporated into the copper matrix. In order to predict the thermal behaviour of $\mathrm{Cu} / \mathrm{CNTs}$ composites using ANSYS software, thermal conductivities of these composites must be known (inputted data). Three different methods have been adopted to calculate the thermal conductivities of $\mathrm{Cu} / \mathrm{CNTs}$ composites. These methods were explained briefly below:

\subsubsection{Rules of Mixtures}

Rules of mixtures are mathematical expressions which give some property of the composite in terms of the properties, quantity and arrangement of its constituents. Assuming that CNTs have a unidirectional dispersion in the $\mathrm{Cu}$ matrix and parallel to heat flow

\subsubsection{Mathematical Prediction Model by Nan et al. (2003)}

Several theoretical models have been proposed base on effective medium approach (EMA) of Maxwell-Garnett and take the linear nature of the CNTs in account. Nan et al. (2003) have proposed a simple model for predicting the effective thermal conductivity of CNTs-reinforced composites in terms of a Maxwell-Garnett effective medium theory. Assuming a random dispersion of carbon nanotubes and high aspect ratio where $\mathrm{p}>1000$.

The effective thermal conductivity, Ke, of the composite is given by [7]:

$$
k_{e} / k_{m}=1+f p / 3\left(k_{c} / k_{m}\right)
$$


Where, the subscripts e, $\mathrm{m}$ and $\mathrm{c}$ are referring to composite, copper matrix and CNTs, respectively.

\subsubsection{Mathematical Prediction Model by Nan et al. (2004)}

In 2004, Nan and co-workers have further modified their theory by incorporating the influence of interface thermal resistance and suggested the following relation [8]:

$$
k_{\varepsilon} / k_{m}=1+f p / 3\left(\frac{k_{\varepsilon} / k_{m}}{p+\left({ }^{\left.2 R_{k} / d\right) \times\left(k_{c} / k_{m}\right)}\right)}\right)
$$

Where, $R_{k}$ is the interfacial resistance between the carbon nanotube and matrix. $d$ is the diameter of carbon nanotube. In the calculations, the interfacial resistance value, $\mathrm{R}_{\mathrm{k}}=8.3 \times 10^{-8} \mathrm{~m}^{2} \mathrm{~K} / \mathrm{W}$. This value was experimentally determined by Huxtable et al. [11]. The thermal conductivities of copper and carbon nanotube are assumed to be 390 and $3000 \mathrm{~W} / \mathrm{m} . \mathrm{K}$ [12], respectively.

In the present study, CNTs reinforced $\mathrm{Cu}$ matrices with different contents of CNTs $(2,5,7.5$ and 10) were investigated and their thermal conductivity measured experimentally and evaluated numerically using the rule of mixtures, Nan's model (2003) and Nan's model (2004).

\section{Results and Discussion}

\subsection{Thermal conductivity Prediction of Cu/CNTs Composites}

The comparison between different thermal conductivities measurements of $\mathrm{Cu} / \mathrm{CNT}$ s nanocomposites such experimental data, the rule of mixtures and the theoretical prediction models is presented in Figure 2. It can be observe that thermal conductivities of the composites measured using rule of mixture showed higher values compared to those predicted by other theoretical models. On the other hand, the theoretical prediction without considering the interface thermal resistance gives the pronounced over-estimation confirming the large impact of interface thermal resistance. The modified model, which includes the interface thermal resistance, therefore provides a preferable estimate for predicting the thermal conductivity of $0-10$ vol.\% $\mathrm{Cu} / \mathrm{CNTs}$ composites. The corresponding small deviations are basically attributed to the non-straightness and interactions of the CNTs, which are not considered in theoretical prediction. Besides, actually grown nanotubes contained surface defects or catalyst particles that could also scatter the heat flow.

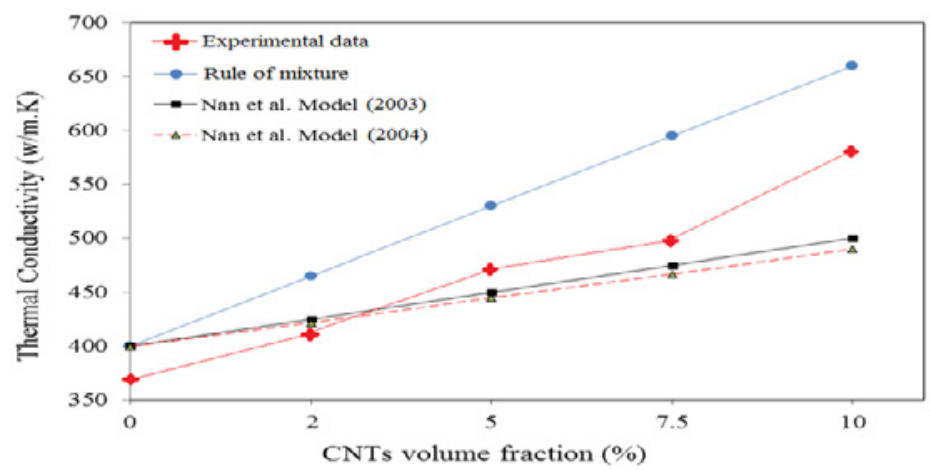

Figure 2. A comparison between the experimentally measured thermal conductivity of $\mathrm{Cu} / \mathrm{CNTs}$ composites with different theoretical prediction models 


\section{Conclusions}

Thermal behaviour of heat sink nanocomposites made of copper reinforced by carbon nanotubes up to 10 Vol. \% was predicted using finite element method. The results showed that rules of mixture method have recorded the highest heat flux distribution for the all composites. In contrast, Nan et al. prediction model which takes into account the influence of the interface thermal resistance between CNTs and copper particles, has shown the lowest thermal conductivity which considered as the closest results to the experimental measurement. The experimentally measured thermal conductivities showed remarkable increase after adding 5 vol.\% CNTs and higher than the thermal conductivities predicted via Nan models, indicating that the improved fabrication technique of powder injection molding that has been used to produced $\mathrm{Cu} / \mathrm{CNTs}$ nanocomposites has overcome the challenges assumed in the mathematical models.

\section{Acknowledgment}

Authors would like to thank Universiti Teknologi PETRONAS for supporting this research and providing the software simulation program.

\section{References}

1. J. Barcena, J.M., J. Coleto and I. Obieta, Novel Copper/Carbon Nanofibres Composites for High Thermal Conductivity Electronic Packaging, (2009)

2. Dai, H., Carbon nanotubes: opportunities and challenges. Surface Science, (2002)

3. Erik T. Thostensona, Z.R., Tsu-Wei Choua, Composites Science and Technology, (2001)

4. George, K. T. K., R. Rahul, S. Yamdagni, Scripta Materialia, (2005)

5. Kwon, Hansang, Mehdi Estili, Kenta Takagi, Takamichi Miyazaki, and Akira Kawasaki. Carbon 47 (3), 570-577 (2009)

6. Song, Young Seok, and Jae Ryoun Youn. "Evaluation of effective thermal conductivity for carbon nanotube/polymer composites using control volume finite element method." Carbon 44 (4) 710-717 (2006)

7. C. W. Nan, Z.S., Y. Lin, Chemical Physics Letters 375, 2003.

8. Nan Cw, L.G., Lin YH, Li M.., Appl Phys Lett, 85, 3549-3551 (2004)

9. Ali S. Muhsan, Faiz Ahmad, Norani M. Mohamed, and M. Rafi Raza, Journal of Nanoengineering and Nanomanufacturing 3.3 248-252 (2013)

10. Ali S. Muhsan, Faiz Ahmad, Norani M. Mohamed, Putri S.M.BT M. Yusoff and M.R. Raza, Journal of Applied Sciences, 12, 2397-2403 (2012)

11. Xiangqiao Yan, et al. Comput Mech 36 76-82(2005)

12. Huxtable, S. T., Cahill, D. G., Shenogin, S., Xue, L., Ozisik, R., Barone, P., Usrey, M., Strano, M. S., Siddons, G., Shim, M., Keblinski, P., Nat. Mat., 2 (11), 731-734 (2003) 